Santa Clara University

Scholar Commons

Physics

College of Arts \& Sciences

10-1-1993

\title{
Low-field magnetoresistance in granular Pb films near the insulator-superconductor transition
}

Richard P. Barber Jr.

SantaClara University, rbarber@scu.edu

Robert C. Dynes

Follow this and additional works at: http://scholarcommons.scu.edu/physics

Part of the Physics Commons

\section{Recommended Citation}

R. P. Barber, Jr. and R. C. Dynes, "Low-field magnetoresistance in $\mathrm{Pb}$ films near the insulator-superconductor transition," (contributed talk) presented at the March Meeting of the American Physical Society, Seattle, 1993; Bulletin of the American Physical Society, 38(1), 526, (1993). 


\title{
Low-field magnetoresistance in granular $\mathrm{Pb}$ films near the insulator-superconductor transition
}

\author{
R. P. Barber, Jr. and R. C. Dynes \\ Department of Physics, University of California, San Diego, La Jolla, California 92093-0319
}

(Received 29 July 1993)

\begin{abstract}
We have studied the insulator-superconductor transition in quench-condensed granular $\mathrm{Pb}$ films from 0.1 to $10 \mathrm{~K}$. Resistance measurements were made in zero magnetic field and low noise conditions. Magnetoresistance measurements were also performed for low magnetic fields (less than $100 \mathrm{G}$ ). The magnetoresistance results on the superconducting side of the transition suggest that we are directly probing the finite length scales associated with the range of phase coherence in these granular materials.
\end{abstract}

Studies of the insulator-superconductor transition in thin metal films have shown that superconductivity is destroyed in two ways depending on the morphology of the sample. ${ }^{1-7}$ In the case of uniform films deposited onto amorphous Ge underlayers, the amplitude of the order parameter, $\Delta$, decreases with decreasing film thickness until the pair wave function, $\Delta^{1 / 2} e^{i \phi}$, vanishes and the film becomes insulating. $5,6 T_{c}$ also decreases in such a way that the coupling, $2 \Delta / k T_{c}$, has been observed to remain constant over nearly 2 orders of magnitude. ${ }^{5}$ For samples deposited onto clean insulating substrates (no Ge underlayer), the structure is apparently granular, and superconductivity is destroyed by the breakdown of longrange phase coherence across the sample. ${ }^{1-4} T_{c}$ for the individual grains is essentially constant throughout the insulator-superconductor transition. We report on zero magnetic-field electrical transport studies of granular $\mathrm{Pb}$ films from $100 \mathrm{mK}$ to $10 \mathrm{~K}$ and the magnetoresistance of these films for low fields. We believe that these measurements directly probe length scales associated with longrange phase coherence on the superconducting side of the transition.

Thin film samples are prepared by evaporating $\mathrm{Pb}$ from a thermal source onto a fire-polished glass substrate held at liquid-helium temperatures. The substrate is thermally connected to the mixing chamber of a ${ }^{3} \mathrm{He}-{ }^{4} \mathrm{He}$ dilution refrigerator. This arrangement allows for temperature control between 0.08 and $10 \mathrm{~K}$. The dilution refrigerator is contained in an exchange gas can with the evaporation source mounted opposite the substrate. The exchange gas can is completely immersed in a liquid-helium bath so that all measurements are performed under ultrahigh vacuum conditions. A quartz crystal microbalance is used to monitor the depositions. The substrate has preevaporated Au contacts $(<100 \AA$ thick) to accommodate four-wire electrical transport measurements. Voltage probes on two additional contiguous regions of the sample allow for measurements to check for macroscopic uniformity. The three adjacent regions are typically within $10 \%$ of the same sheet resistance indicating good uniformity. ac lock-in resistance measurement and dc current-voltage $(I-V)$ measurement techniques are used. The sample is positioned in a $5 \mathrm{~T}$ superconducting magnet such that $H$ is perpendicular to the film to allow the study of the effects of magnetic field. The entire ap- paratus is enclosed in a shielded room to minimize external noise.

During the deposition of the film, the substrate is held at $10 \mathrm{~K}$. This temperature ensures that the $\mathrm{Pb}$ will exhibit only normal-state properties and greatly simplifies control of the evaporation. Because there is a threshold average thickness before appreciable conductance $\left(G>10^{-9} \mathrm{~S}\right)$ is measured, the films are apparently granular. This initial deposition corresponds to an amount of $\mathrm{Pb}$ equivalent to a 90-100 $\AA$ uniform film. We terminate this first deposition when the sheet resistance of the film, $R_{\square}$, is roughly $1-10 \times 10^{6} \Omega$. The film sheet resistance versus temperature, $R_{\square}(T)$, is then measured. The resistance values are obtained from the slopes of the $I-V$ curves in the low bias regime about zero voltage (typically from minus to plus $10-100 \mathrm{nV}$ ). Care is taken to prevent warming the film above $10 \mathrm{~K}$, since this causes irreversible annealing of the sample. ${ }^{4}$ Figure 1 shows zero magnetic field $R_{\square}(T)$ for a typical series of depositions. Note that we have plotted $\log _{10}(R)$ since the data covers almost 12 orders of magnitude in resistance. For a normal-state sheet resistance, $R_{\square}(10 \mathrm{~K})$, of several megaohms (curve $a$ ), the sample is clearly insulating $(R \rightarrow \infty$ as $T \rightarrow 0)$. As temperature is lowered $R_{\square}(T)$ rises to very large values $\left(R>10^{9} \Omega\right)$. With further deposition at these high resistances, the normal-state resistance decreases precipitously with increasing film thickness. An average increment of a fraction of an angstrom causes a change typical of the one from curve $a$ to curve $b$. The film is still insulating at low $T$ showing activation behavior but with lower activation energy. Consequently we can measure $R_{\square}(T)$ to a somewhat lower $T$. At a slightly greater thickness (curve $c$ ), $R_{\square}(T)$ is no longer monotonically increasing as we lower $T$. Below a temperature corresponding to roughly the $T_{c}$ for bulk $\mathrm{Pb}$, $R_{\square}(T)$ first decreases before increasing at lower temperatures finally showing a tendency for insulating behavior at the lowest temperatures. Upon further deposition there is a remarkable change to a monotonically decreasing $R_{\square}(T)$ dependence (curve $d$ ). Eventually (curves $e-i), R_{\square}(T)$ appears to evolve towards superconductor transitions albeit with long resistive tails. Indeed it is not clear in curves $d-f$ that the $T=0$ limit is superconducting. At large thicknesses (curve $j$ ), these tails disappear 


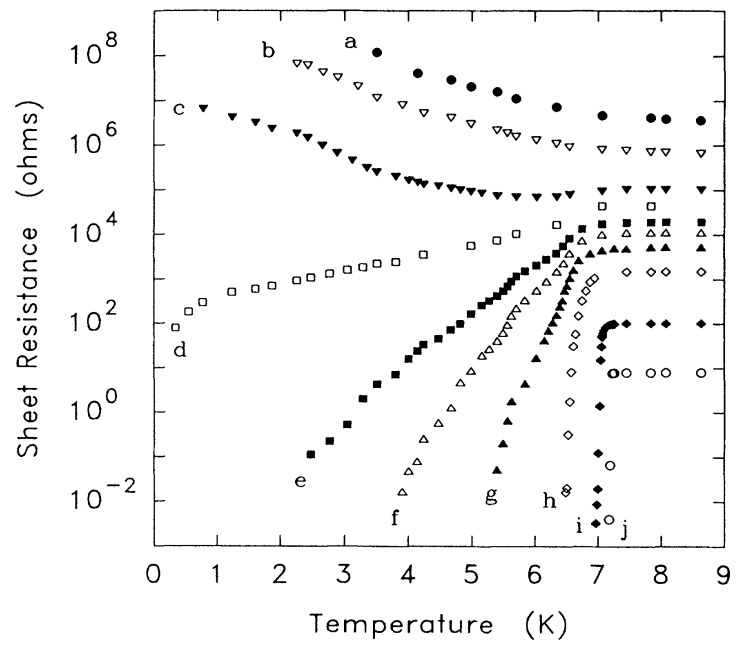

FIG. 1. Sheet resistance vs temperature for a granular $\mathrm{Pb}$ film. Curves $a-j$ represent increasing thickness.

and a sharp transition is observed.

In the lowest temperature data in the resistive tails (e.g., curve $d$ ), there are subtle difficulties in making accurate resistance measurements. The $I-V$ curves in these regions become highly nonlinear except at very low current. As the linear regime becomes vanishingly small (and $R \rightarrow 0$ at zero bias), the effects of external noise and very small magnetic fields become substantial. Noise causes measurements to average over the $I-V$ curve, smearing out the nonlinearities and producing an errant measure of $R$ somewhat higher than the true value. Previous studies ${ }^{1,3,4}$ have not addressed the importance of small magnetic fields which will be shown (e.g., Fig. 2) to have a profound effect on the measured resistance in this regime. Paying attention to these important details, careful measurements showed no evidence of any temperature independent $R_{\square}(T)$. As noise was reduced, field was zeroed, or temperature lowered; resistance was always observed to decrease. A similar effect was seen in data such as that in curve $c$. In that case, careful noise reduction always resulted in resistance increasing as temperature was lowered.

From the data in Fig. 1, we feel very uneasy identifying a superconducting transition temperature $T_{c}$. In studies of the $I-V$ characteristics for these films, we have identified a temperature where the range (current and voltage) over which the $I-V$ curve is linear collapses to zero. It is this point that we identify as indicating a zero resistance $\left(d V /\left.d I\right|_{v=0}=0\right)$ state.

We have found that these data can be drastically modified if proper attention is not paid to the magnetic field on the sample. Small fields strongly affect the resistance. Figure 2 shows representative results of the lowfield magnetoresistance data for a film on the superconducting side of the insulator-superconductor transition (for example, curve $e$ in Fig. 1). The positive magnetoresistance shown can be seen just below the onset of the superconducting transition near $7 \mathrm{~K} . R(H)$ was determined using an ac lock-in resistance measurement while

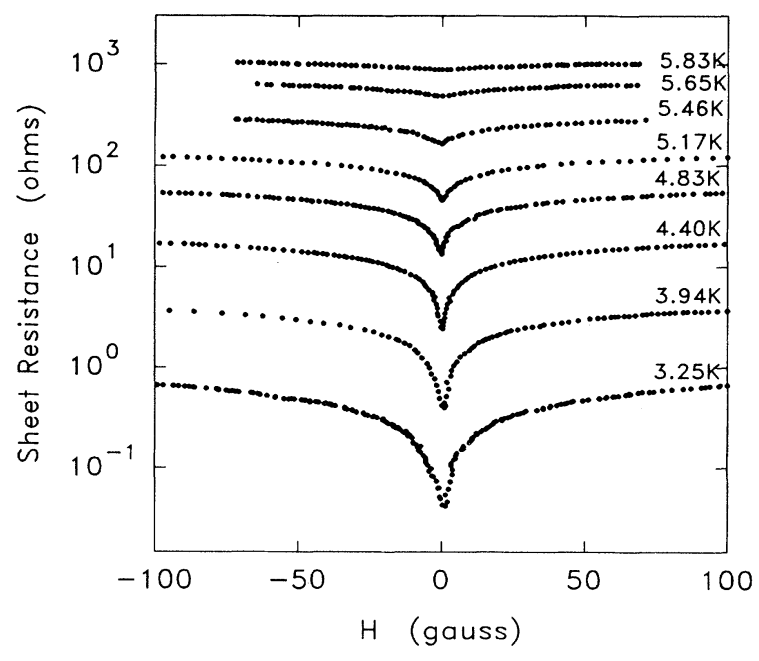

FIG. 2. Magnetoresistance at various temperatures for a film exhibiting a long resistive tail (like curve $e$, Fig. 1).

field was swept slowly. Since Fig. 2 plots $\log _{10}[R(H)]$, the size of the magnetoresistive increase relative to the zero-field resistance at various temperatures can be compared. At field scales of a few tens of gauss it is common to see factors of 20 or more increase in $R(H)$ over $R(0)$ at the lowest temperatures. Though not apparent in this figure, fields of as little as $0.01 \mathrm{G}$ were observed to cause measurable increases in $R$ at the lowest temperatures. At magnetic fields higher than some crossover point that we designate as $H_{x}$, all of the $R(H)$ data is best described by a power-law dependence $\left(R \sim H^{\alpha}\right)$ for intermediate fields $(H<50-100 \mathrm{G})$. At higher fields, the dependence is also consistent with a logarithmic behavior $(R \sim \log H)$. Since we do not have a quantitative theory for this magnetoresistance (a qualitative picture will be discussed below), we choose to display the data on a log-log plot (power-law behavior). Figure 3 shows the power-law dependence and our construction to determine $H_{x}$. The slope of the linear region determines the exponent $\alpha$. We identify $H_{x}$ as the crossover point between power-law dependence of $R(H)$ and the weak dependence of $R(H)$ near zero magnetic field. In Fig. 4 we show $H_{x}$ and $\alpha$ as a function of temperature for the data shown in Fig. 2. Note that we plot $\log _{10}\left(H_{x}\right)$ vs temperature. The fact that this data falls roughly on a straight line indicates that $H_{x}$ increases exponentially with temperature. $\alpha$ also has a linear dependence on temperature. It should be noted here that for the range of data presented, a power law best describes the intermediate field results. However, for curves such as the $5.83 \mathrm{~K}$ data, a value for $\alpha=0.07$ means that this form is practically indistinguishable from a logarithmic one.

We physically interpret the crossover field $H_{x}$ in terms of the size of the regions of superconducting phase coherence. In the insulating regime of the $R_{\square}(T)$ data, direct tunneling observations of the superconducting energy gap have been made. ${ }^{8}$ This observation shows that the grains which make up the film are already individual superconductors themselves. However, there is no globally 


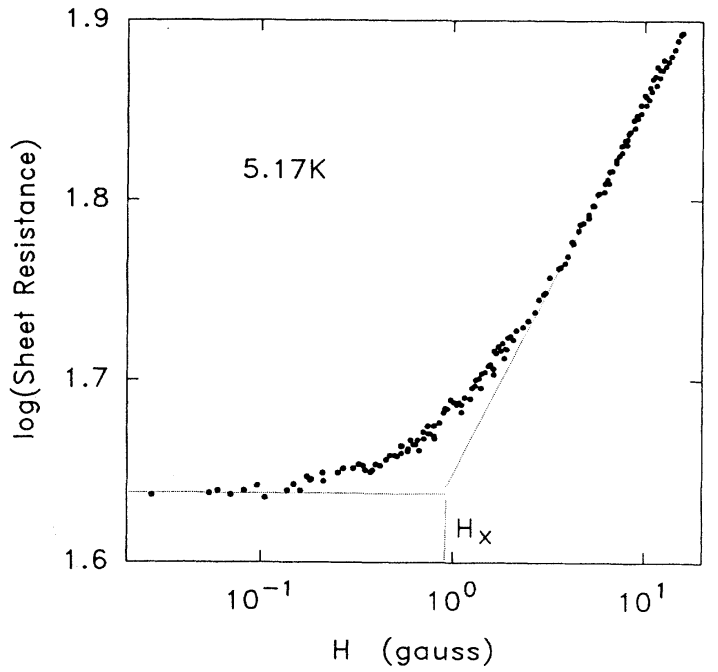

FIG. 3. Sheet resistance vs magnetic field for the $5.17 \mathrm{~K}$ curve of Fig. 2. The plot is on a log-log scale to show the power-law construction for $H_{x}$ and $\alpha$ where $R \sim H^{\alpha}$ for $H>H_{x}$.

coherent phase across the sample. In other words, each of the grains or small clusters of grains have phase coherence but the coherence length is rather short. As the average thickness increases and the coupling between grains improves, larger regions of the sample become coherently connected. On the "superconducting" side of the transition where the long resistive tails occur, increasing thickness means that the phase coupling is stronger so that resistance vanishes below the experimental threshold at progressively higher temperatures. Note that these "tails" are almost linear when plotted as $\log (R)$ indicating a roughly exponential dependence of resistance on temperature.

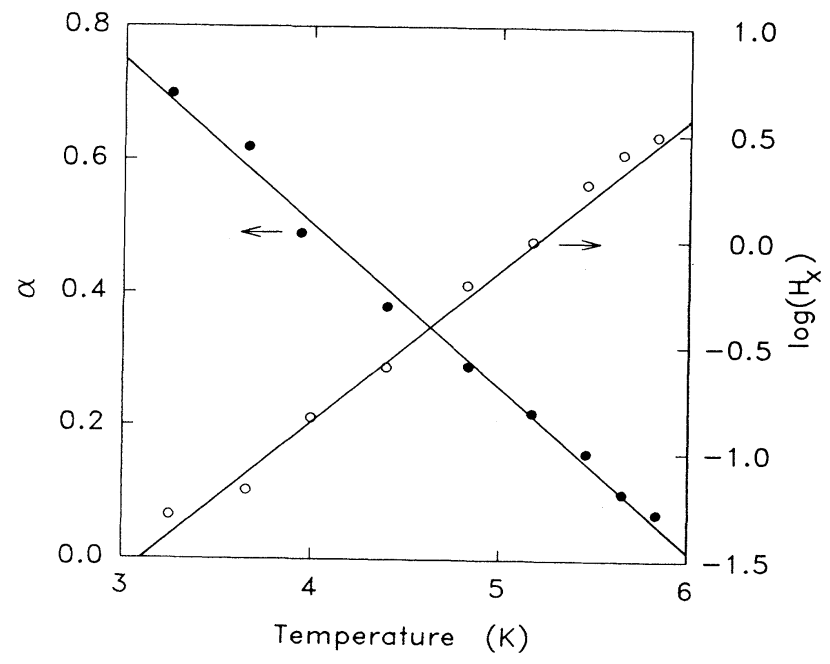

FIG. 4. $\alpha$ and $H_{x}$ vs temperature for the data in Fig. 2. Solid lines are least squares fits showing the linear dependence of $\alpha$ and $\log _{10}\left(H_{x}\right)$ on temperature.
The magnetoresistance data are also consistent with this picture. The size of $H_{x}$ as a function of temperature can be related more directly to a size scale. $A=\Phi_{0} / H_{x}$, where $\Phi_{0}$ is the superconducting flux quantum, $H_{x}$ is the associated magnetic field, and $A$ is some characteristic area. We identify the value of $H_{x}$ as that field where a flux quantum is enclosed by an area roughly the size of the phase coherence. This region is the maximum area over which screening supercurrents can be sustained. For lower fields (larger areas) phase coherence is destroyed and no magnetoresistance results. Larger fields correspond to smaller lengths, and the effects increase first as a power law and then logarithmically with $H$. We draw on a similar picture in weak localization where magnetoresistance measurements are used to determine the phase breaking length $L_{\phi} \cdot{ }^{9}$ In the present experiments, we believe that these measurements allow us to determine the length scale over which there is pair phase coherence (and hence superconductivity). In the same way that a loop geometry shows $h / 2 e$ (AranovAltshuler-Spivak) oscillations if $L_{\phi}$ is greater than the loop size, ${ }^{10}$ a loop of size less than the length scale of the superconducting phase coherence would in our samples show Little-Parks oscillations. As this length becomes longer than the sample size, we expect zero resistance. For $H_{x}=1 \mathrm{G}$ the relevant length scale $l_{\phi} \sim A^{1 / 2}$ $=\left[\Phi_{0} / H_{x}\right]^{1 / 2}=5 \mu \mathrm{m}$. For $H_{x}=0.01 \mathrm{G}$ the length scale becomes $0.05 \mathrm{~mm}$. As $H_{x}$ becomes smaller, the area of coherence increases. Given the temperature dependence of $H_{x}$ in Fig. 4, this means that $A$ grows exponentially as $T$ decreases. In other words the number of regions of differing (uncorrelated) phase is decreasing exponentially as $T$ is lowered. In terms of resistance measured across the film, voltages correspond to changes in the superconducting phase. Therefore fewer regions of differing phase (equivalently larger regions of phase coherence) would imply smaller resistances with a similar functional dependence on temperature; namely exponential. Finally, of course, as the length scale exceeds the size of the sample, the film becomes a zero resistance superconductor. In the case of the sample in Figs. 3 and 4, a simple extrapolation of the $H_{x}$ versus temperature curve would indicate a $T=0$ value for $H_{x}$ which yields a length scale that is still 1-2 orders of magnitude smaller than the sample. Presumably this simple temperature dependence for $H_{x}$ will break down at some point, and $H_{x}$ will decrease $\left(l_{\phi}\right.$ will increase) more rapidly as temperature is lowered. However, measurement in that regime in the present experiments would require $\mu \mathrm{G}$ and $\mu \Omega$ resolution.

This picture of a finite length scale for phase coherence gives a natural explanation for the lower temperature dissipation and the long resistive tails in Fig. 1. An alternative mechanism for dissipation in a highly disordered two-dimensional superconductor is vortex motion. At zero applied field, a disordered superconductor would have equal numbers of positive and negative vortices, some bound and some unbound. For applied currents, the unbound vortices could move causing dissipation. We do not believe that this is an appropriate model in this case. We envision this granular film as a random array of weakly coupled Josephson junctions. The relevant 
length scale for a vortex is the Josephson penetration depth $\lambda_{J}$. A simple calculation of this quantity yields a $\lambda_{J}$ of roughly $0.4 \mathrm{~mm}$, a value comparable to the size of our sample. Vortices cannot be smaller than this, so we do not believe that we can think of this film as supporting positive and negative vortices. We are thus led back to our more microscopic picture of broken phase coherence.

In summary, we have studied electrical transport properties near the insulator-superconductor transition in quench condensed granular $\mathrm{Pb}$ films. The measurements have been made down to $0.1 \mathrm{~K}$ with careful attention to external noise and stray magnetic fields. Magnetoresistance data for small magnetic fields is consistent with the interpretation that we are probing the length scales of long-range phase coherence in this dissipative regime.

We acknowledge valuable discussions with F. Sharifi, J. Valles, S. Kivelson, S. Chakravarty, and R. Ferrell. This work was supported in part by ONR Grant No. N00014-91-5-1320.
${ }^{1}$ R. C. Dynes, J. P. Garno, and J. M. Rowell, Phys. Rev. Lett. 40, 479 (1978); A. E. White, R. C. Dynes, and J. P. Garno, Phys. Rev. B 33, 3349 (1986).

2J. M. Valles, Jr. and R. C. Dynes, in Physical Phenomena in Granular Materials, edited by G. D. Cody, T. H. Geballe, and P. Sheng, MRS Symposia Proceedings No. 195 (Materials Research Society, Pittsburgh, 1990), p. 375.

${ }^{3}$ H. M. Jaeger, D. B. Haviland, B. G. Orr, and A. M. Goldman, Phys. Rev. B 40, 182 (1989), and references within.

${ }^{4}$ R. P. Barber, Jr. and R. E. Glover III, Phys. Rev. B 42, 6754 (1990).

5J. M. Valles, Jr., R. C. Dynes, and J. P. Garno, Phys. Rev.
Lett. 69, 3567 (1992).

${ }^{6}$ R. C. Dynes, A. E. White, J. M. Graybeal, and J. P. Garno, Phys. Rev. Lett. 57, 2195 (1986).

${ }^{7}$ D. B. Haviland, Y. Liu, and A. M. Goldman, Phys. Rev. Lett. 62, 2180 (1989).

${ }^{8}$ R. P. Barber, Jr., L. M. Merchant, A. La Porta, and R. C. Dynes (unpublished).

${ }^{9}$ D. J. Bishop, R. C. Dynes, and D. C. Tsui, Phys. Rev. B 26, 773 (1982).

${ }^{10}$ R. A. Webb, S. Washburn, C. P. Umbach, and R. P. Laibowitz, Phys. Rev. Lett. 54, 2696 (1985). 